ITEP-LAT/2003-10

KANAZAWA-03-14

\title{
GLUODYNAMICS IN EXTERNAL FIELD: A TEST OF THE DUAL SUPERCONDUCTOR PICTURE $*, \dagger$
}

\author{
M. N. CHERNODUB \\ ITP, Kanazawa University, Kanazawa, 920-1192, Japan \\ ITEP, B. Cheremushkinskaya 25, Moscow, 117259, Russia
}

\begin{abstract}
We study gluodynamics in an external Abelian electromagnetic field within the dual superconductor approach. We show that the $S U(2)$ gluodynamics should possess a deconfining phase transition at zero temperature at certain value of the external field. A dual superconductor model for the $S U(3)$ gauge theory in external field predicts a rich phase structure containing confinement, asymmetric confinement and deconfinement phases. These results can be used to check the validity of the dual superconductor description of gluodynamics in external fields. We also discuss the gauge-independence of the obtained results.
\end{abstract}

1. We discuss the properties of the $S U(2)$ and $S U(3)$ gluodynamics in the external electromagnetic field using the dual superconductor approach ${ }^{1}$ based on the Abelian monopole condensation. The condensate - observed ${ }^{2}$ in various numerical simulations at low temperatures- forces a chromoelectric flux coming from (anti-)quarks to squeeze into a confining string. This picture of confinement has been confirmed in various lattice simulations ${ }^{3}$.

A common feature of known superconductors is that at strong enough magnetic fields the superconductivity is destroyed and the superconductor goes in the normal (metal) state. In the dual superconductor model a similar effect ${ }^{4}$ leads to the deconfinement phase transition. Below we study the phase diagram of gluodynamics within the dual superconductor approach. We work in the Bogomol'ny limit ${ }^{5}$ supported by lattice simulations ${ }^{6}$.

2. Let us first consider the $S U(2)$ gauge theory in the $4 D$ Euclidean space. The infrared properties of the vacuum of this model can be described by the Abelian Higgs (or, Ginzburg-Landau) Lagrangian:

$$
L_{G L}[B, \Phi]=\frac{1}{4} F_{\mu \nu}^{2}+\frac{1}{2}\left|D_{\mu}(B) \Phi\right|^{2}+\lambda\left(|\Phi|^{2}-\eta^{2}\right)^{2},
$$

\footnotetext{
*Talk given at "Confinement 2003", RIKEN, Wako, Japan, 21-24 July 2003.
}

${ }^{\dagger}$ This work is supported by the JSPS Fellowship No. P01023. 
where $F_{\mu \nu}=\partial_{\mu} B_{\nu}-\partial_{\nu} B_{\mu}$ is the field strength of the dual gauge field $B_{\mu}, \Phi$ is the monopole field with the magnetic charge $g_{M}$, and $D_{\mu}=\partial_{\mu}+i g_{M} B_{\mu}$ is the covariant derivative. The gauge field $B_{\mu}$ is dual to the third component of the gluon field in an Abelian gauge. The model possesses the dual $U(1)$ gauge symmetry, $B_{\mu} \rightarrow B_{\mu}-\partial_{\mu} \alpha, \Phi \rightarrow e^{i g_{M} \alpha} \Phi$. The form of the potential implies the existence of the monopole condensate, $|\langle\Phi\rangle|=\eta>0$.

Consider the four-dimensional sample of the (dual) superconductor occupying half-space, $x_{2} \geqslant 0$. Let us apply the constant external EM field $F_{\mu \nu}^{\mathrm{ext}}=\varepsilon_{\mu \nu 34} H^{\mathrm{ext}}$ to the boundary of the superconductor. The external field is screened due to the induced superconducting current,

$$
J_{\mu}=\Im m\left(\Phi^{*} D_{\mu}(B) \Phi\right) \equiv|\Phi|^{2} \cdot v_{\mu}, \quad v_{\mu}=\partial_{\mu} \varphi+g_{M} B_{\mu},
$$

where we have set $\Phi=|\Phi| e^{i \varphi}$. The current is parallel to the boundary of the superconductor. The monopole kinetic term in Eq. (1) can be written as $\left|D_{\mu} \Phi\right|^{2}=\left(\partial_{\mu}|\Phi|\right)^{2}+|\Phi|^{2} v_{\mu}^{2}$. Clearly, a non-zero current provides an additional positively-defined term in the Lagrangian $\left(\propto|\Phi|^{2}\right)$. As a result, the external field destroys the monopole condensate.

We treat the model (1) classically. It is convenient to rewrite the action of the model (1) as an integral in a two-dimensional plane. One of the directions of the plane is the depth of the dual superconductor, $x_{2}$, while the second is given by the direction of the current (2). Choosing $J_{\mu} \propto \delta_{\mu, 1}$ the first two terms of eq. (1) become, respectively, $F_{\mu \nu}^{2} / 4=H^{2} / 2$ and

$$
\left|D_{\mu} \Phi\right|^{2}=\sum_{\alpha=1,2}\left|D_{\alpha} \Phi\right|^{2}=\left|\left(D_{1} \pm i D_{2}\right) \Phi\right|^{2} \mp 2 \varepsilon_{\alpha \beta} \partial_{\alpha} J_{\beta} \mp g_{M} H|\Phi|^{2} .
$$

In the Bogomol'ny limit, $g_{M}^{2} / \lambda=8$, the action of the model is:

$$
S=\frac{L_{3} L_{4}}{2} \int \mathrm{d}^{2} x\left\{\left|\left(D_{1}-i D_{2}\right) \Phi\right|^{2}+\left[H+\frac{g_{M}}{2}\left(|\Phi|^{2}-\eta^{2}\right)\right]^{2}\right\}+S_{f}+S_{J},(4)
$$

where $L_{i}$ is the (infinitely large) length of the dual superconductor in $i^{\text {th }}$ direction, $S_{J}=L_{3} L_{4} \int \mathrm{d}^{2} x \varepsilon_{\alpha \beta} \partial_{\alpha} J_{\beta}=L_{1} L_{3} L_{4} J_{1}\left(x_{2}=0\right)$ is the action of the surface current and $S_{f}=g_{M} L_{3} L_{4} \eta^{2} \int \mathrm{d}^{2} x H / 2$ is the "flux" action.

The Bogomol'ny equations minimize the action (4),

$$
\left(D_{1}-D_{2}\right) \Phi=0, \quad H+\frac{g_{M}}{2}\left(|\Phi|^{2}-\eta^{2}\right)=0 .
$$

The second equation gives the monopole condensate at the boundary, $\left|\Phi\left(x_{2}=0\right)\right|^{2}=\eta^{2}-2 H^{e x t} / g_{M}$. The condensate disappears at the critical value of the external EM field, $H^{\text {ext }}=H_{\text {cr }}=g_{M} \eta^{2} / 2$. 
At zero temperature and in the absence of the external fields the tension of the string spanned on trajectories of the fundamental charges can be evaluated exactly in the Bogomol'ny limit ${ }^{5,7}, \sigma=\pi \eta^{2}$. Using the Dirac relation between magnetic $\left(g_{M}\right)$ and electric $(g)$ charges, $g_{M} g=2 \pi$, we get the exact value of the critical EM field in terms of the string tension:

$$
g H_{\mathrm{cr}} / \sigma=1, \quad \text { for } \mathrm{SU}(2) .
$$

The dual superconductivity is destroyed in the bulk if $H \geqslant H_{\mathrm{cr}}$.

3. Now let us consider the Lagrangian of the $[U(1)]^{2}$ dual superconductor model corresponding to $S U(3)$ gluodynamics ${ }^{8}$ :

$$
L=\frac{1}{4} F_{\mu \nu}^{a} F^{a, \mu \nu}+\sum_{i=1}^{3}\left[\frac{1}{2}\left|\left(\partial_{\mu}+i g_{M} \varepsilon_{i}^{a} B_{\mu}^{a}\right) \Phi_{i}\right|^{2}+\lambda\left(\left|\Phi_{i}\right|^{2}-\eta^{2}\right)^{2}\right],
$$

where $F_{\mu \nu}^{a}=\partial_{\mu} B_{\nu}^{a}-\partial_{\nu} B_{\mu}^{a}$ is the field strength of the gauge field $B_{\mu}^{a}$, $a=3,8$. The phases of the monopole fields, $\Phi_{i}, i=1,2,3$, satisfy the relation $\sum_{i=1}^{3} \arg \Phi_{i}=0$. The $\epsilon$-symbols are the root vectors of the $S U(3)$ group: $\vec{\epsilon}_{1}=(1,0), \vec{\epsilon}_{2}=(-1 / 2,-\sqrt{3} / 2), \vec{\epsilon}_{3}=(-1 / 2, \sqrt{3} / 2)$. In Eq. (6) no summation over the Latin index $i$ is implied. The gauge fields $B_{\mu}^{3,8}$ are dual to the diagonal components $a=3,8$ of the gluon field $A_{\mu}^{a}$. Lagrangian (6) respects the dual $[U(1)]^{2}$ gauge invariance: $B_{\mu}^{a} \rightarrow B_{\mu}^{a}+\partial_{\mu} \alpha^{a}, \theta_{i} \rightarrow \theta_{i}+$ $g_{M}\left(\varepsilon_{i}^{3} \alpha^{3}+\varepsilon_{i}^{8} \alpha^{8}\right), a=3,8, i=1,2,3$, where $\alpha^{3}$ and $\alpha^{8}$ are the parameters of the gauge transformation.

In the $[U(1)]^{2}$ Bogomol'ny limit, $g_{M}^{2} / \lambda=16 / 3$, the equations of motion

$$
\left(D_{1}^{(i)} \pm i D_{2}^{(i)}\right) \chi_{i}=0, \quad H^{(i)} \mp \frac{3 g_{M}}{4}\left(\left|\chi_{i}\right|^{2}-\eta^{2}\right)=0 ; \quad i=1,2,3,
$$

give the same (due to the Weyl symmetry ${ }^{9,10}$ ) critical value for all components of the EM field, $H^{(i)}=\sum_{a=3,8} \epsilon_{i}^{a} H^{a}, i=1,2,3$ :

$$
g H_{\mathrm{cr}}^{(i)} \equiv g \tilde{H}_{c r} / \sigma=3 / 4, \quad \text { for } \mathrm{SU}(3) .
$$

Here we used the Dirac condition and the relation for the fundamental string tension, $\sigma=2 \pi \eta^{2}$, valid in the Bogomol'ny limit of the $[U(1)]^{2}$ model ${ }^{11,9}$. When the strength of the EM component $H^{(i)}$ reaches the $\tilde{H}_{c r}$ value then the monopole field, $\Phi_{i}$, vanishes. Expressing the auxiliary fields $H^{(i)}$ in terms of the components the EM field, $H^{3,8}$, and using Eqs.(8) we get the phase diagram of the $[U(1)]^{2}$ dual superconductor depicted in Figure 1.

The phase diagram contains confinement $(C)$, deconfinement $(D)$ and the asymmetric confinement phases $(A)$. The phase transition depends not 


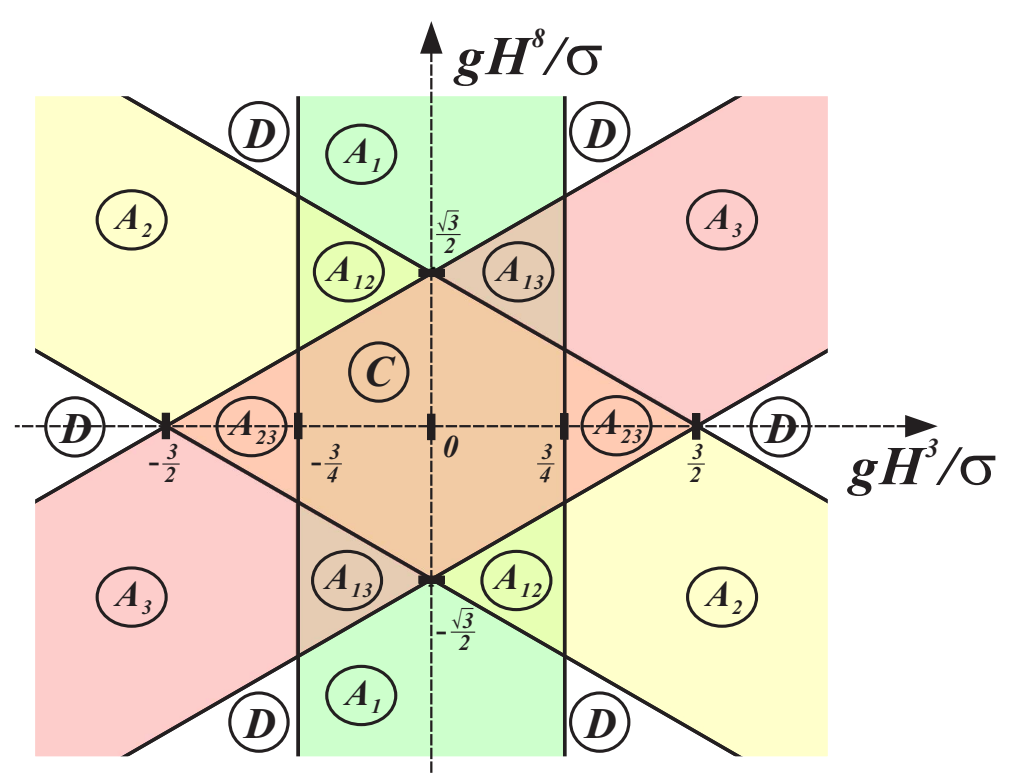

Figure 1. The zero temperature phase diagram of the dual $[U(1)]^{2}$ Higgs model in the Bogomol'ny limit in the presence of the external electromagnetic field.

only on the absolute value of the EM field but also on the ("color") orientation of this field in the Cartan subgroup. At low values of the field the model is always confining regardless of the color orientation. However, as the absolute value of the field is increased, the model enters - depending on color orientation - one of six $\left(A_{12}, A_{13}, A_{23}, A_{1}, A_{2}\right.$ or $\left.A_{3}\right)$ asymmetric confinement phases. In the $A_{i j}$ phase the $i$ th and $j$ th components of the monopole field are condensed while the expectation value of the third component is zero. In the phase $A_{i}$ the $i$ th component is condensed while the others two components are not. With further increase of the field the model either enters the deconfinement phase, $D$, or stays in one of the three asymmetric confinement phases, $A_{1}, A_{2}$ or $A_{3}$.

One can show ${ }^{4}$ that three of six asymmetric confinement phases $\left(A_{12}\right.$, $A_{13}$ and $\left.A_{23}\right)$ contain one baryon and three meson states. These phases are confining since the quarks of all three colors are confined. The other three asymmetric confinement phases $\left(A_{1}, A_{2}\right.$ and $\left.A_{3}\right)$ contain only two light meson states while the baryon bound state is absent.

4. Our results were obtained for the Abelian external fields which are 
applied to the dual superconductor corresponding to a fixed Abelian projection $^{\mathrm{a}}$. Although the fact of the monopole condensation seems to be projection-independent ${ }^{2}$, important properties of the dual superconductor may depend on the on chosen Abelian projection ${ }^{12, \mathrm{~b}}$.

We propose to investigate numerically the phase diagrams of the $S U(2)$ and $S U(3)$ gluodynamics in the Maximal Abelian projection and compare them with the predictions of Ref. ${ }^{4}$. This comparison should reveal whether the dual superconductor picture works at strong external fields or not.

\section{References}

1. Y. Nambu, Phys. Rev. D10 (1974) 4262; G. 't Hooft, in High Energy Physics, ed. A. Zichichi, EPS International Conference, Palermo (1975); S. Mandelstam, Phys. Rept. 23, 245 (1976).

2. M. N. Chernodub, M. I. Polikarpov, A. I. Veselov, JETP Lett. 63 (1996) 411; Phys. Lett. B399 (1997) 267; A. Di Giacomo, G. Paffuti Phys. Rev. D 56 (1997) 6816; N. Nakamura et al, Nucl. Phys. Proc. Suppl. 53 (1997) 512.

3. For a review see, e.g., M. N. Chernodub and M. I. Polikarpov, in "Confinement, Duality and Non-perturbative Aspects of QCD", p.387, Plenum Press, 1998, hep-th/9710205; R. W. Haymaker, Phys. Rept. 315, 153 (1999).

4. M. N. Chernodub, Phys. Lett. B549, 146 (2002).

5. E. B. Bogomolny, Sov.J.Nucl.Phys. 24 (1976) 449 [Yad.Fiz. 24 (1976) 861].

6. M. Baker, J.S. Ball, F. Zachariasen, Phys. Rev. D31 (1985) 2575; ibid. 41 (1990) 2612; S. Maedan, Y. Matsubara, T. Suzuki, Prog. Theor. Phys. 84 (1990) 130; V. Singh, D.A. Browne, R.W. Haymaker, Phys. Lett. B 306, 115 (1993); Y.Matsubara, S. Ejiri, T. Suzuki, Nucl. Phys. Proc. Suppl. 34 (1994) 176; G.S. Bali, C.Schlichter, K.Schilling, Prog. Theor.Phys. Suppl. 131 (1998) 645.

7. H. J. de Vega, F. A. Schaposnik, Phys. Rev. D14 (1976) 1100.

8. S. Maedan and T. Suzuki, Prog. Theor. Phys. 81 (1989) 229.

9. Y. Koma and H. Toki, Phys. Rev. D 62 (2000) 054027;

10. Y. Koma, E. M. Ilgenfritz, T. Suzuki, H. Toki, Phys.Rev. D 64 (2001) 014015.

11. M. N. Chernodub, Phys. Lett. B 474 (2000) 73.

12. M. N. Chernodub, M. I. Polikarpov and A. I. Veselov, Phys. Lett. B 342 (1995) 303; S. Fujimoto, S. Kato, T. Suzuki and T. Tsunemi, Prog. Theor. Phys. Suppl. 138 (2000) 36; J. M. Carmona, M. D'Elia, A. Di Giacomo, B. Lucini and G. Paffuti, Phys. Rev. D 64 (2001) 114507.

13. P. Cea, L. Cosmai, JHEP 0302 (2003) 031; Phys.Rev. D60 (1999) 094506.

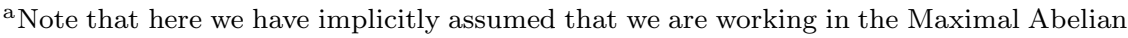
projection where the Bogomol'ny limit is realized ${ }^{6}$.

${ }^{\mathrm{b}}$ In particular, this implies that results of Refs. ${ }^{13}$ for the phase diagram in the external Abelian fields can not be compared with our predictions. 